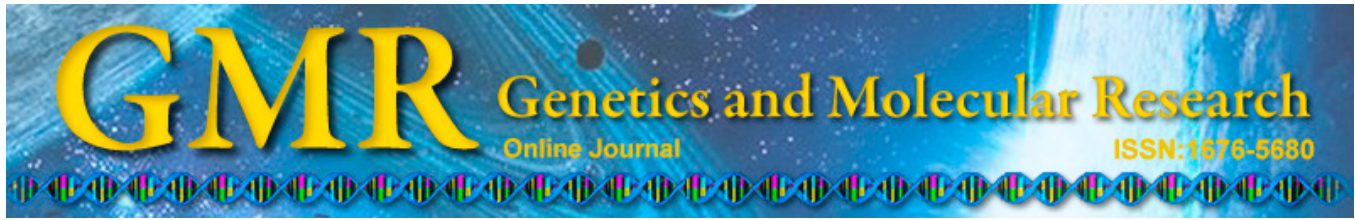

\title{
Predictive potential role of glutathione $S$-transferase polymorphisms in the prognosis of breast cancer
}

\author{
X. Wang ${ }^{1,2}$ and Z.H. Huang ${ }^{1}$ \\ ${ }^{1}$ Department of General Surgery, Zhujiang Hospital, Southern Medical University, \\ Guangzhou, China \\ ${ }^{2}$ Department of General Surgery, The Affiliated Hospital of Inner Mongolia \\ Medical University, Hohhot, China \\ Corresponding author: Z.G. Huang \\ E-mail: zhhuang1999@126.com
}

Genet. Mol. Res. 14 (3): 10236-10241 (2015)

Received January 13, 2015

Accepted June 22, 2015

Published August 28, 2015

DOI http://dx.doi.org/10.4238/2015.August.28.7

\begin{abstract}
The current study aimed at evaluating the association between GSTM1 null/present, GSTT1 null/present, and GSTP1 IIe105Val polymorphisms and clinical response to chemotherapy and treatment outcome of breast cancers patients. Genotyping of GSTP1 rs1695, GSTT1 deletion, and GSTM1 deletion was performed by Polymerase Chain Reaction Restriction Fragment Length Polymorphism (PCR-RFLP) assay. We found that patients with GG genotype of GSTP1 IIe105Val and null genotype of GSTM1 were more likely to have a poorer response to chemotherapy than homozygotes of the most frequent genotype; the ORs $(95 \% \mathrm{CI})$ were $0.37(0.18-0.74)$ and 0.59(0.36-0.97), respectively. By the Cox proportional hazards model, patients with the GG genotype of GSTP1 IIe105Val and null genotype of GSTM1 were found to be correlated with shorter overall survival of breast cancer; the adjusted HR $(95 \% \mathrm{CI})$ were $2.51(1.17-5.32)$ and $2.00(1.15-3.48)$, respectively. Thus, our findings provided statistical evidence that the variants of GSTP1 and GSTM1 polymorphisms could
\end{abstract}


influence the response to chemotherapy and overall survival in breast cancer patients treated with chemotherapy.

Key words: Glutathione $S$-transferases; Polymorphism; Breast cancer

\section{INTRODUCTION}

Breast cancer is by far the most frequent cancer mortality in women, with $\sim 1.67$ million new cases estimated to have occurred in 2012, making it the second most common malignancy among females worldwide (10.9\% of all cancers) (IARC, 2012). Chemotherapy is retained as the standard therapeutic strategies for breast cancer patients, especially those with metastastic settings. Although both these active chemotherapeutic agents are available for many years, the inter-individual efficacy and toxicities still obviously exist among patients with similar disease. Therefore, identifying the molecular biomarkers of drug metabolism enzymes may influence the chemotherapeutic outcomes and toxicity.

The GST enzymes play an important role in the cellular defense system (Strange and Fryer, 1999). The GST enzymes could detoxify chemotherapeutic drugs through their conjugation with glutathione. Further, GSTs gene polymorphisms could reduce the effectiveness of detoxifying cytotoxins generated by chemotherapeutic agents for breast cancer (Townsend and Tew, 2002).

In humans, four major subfamilies of GSTs can be identified, including GSTa, GST $\mu$, GST $\theta$ and GST $\pi$. Each of GSTs is composed of several members, some of which present genetic polymorphisms. Homozygozity of GSTM1 and GSTT1 is member of GST $\mu$ and GST $\theta$, and it leads to absence of phenotypic enzyme activity. The polymorphism of GSTP1 at codon 105 (IIe105Val) is a member of GST $\pi$ subfamily, and shows different catalytic activities (Mishra et al., 2011).

Thus far, many studies have assessed the role of GSTs gene polymorphisms in the response to chemotherapy for breast cancer; however, the results obtained from these studies are inconsistent (Mishra et al., 2011; Duggan et al., 2013; Tulsyan et al., 2013; Oliveira et al., 2010, 2014). Consequently, the current study aimed at evaluating the association between GSTM1 null/present, GSTT1 null/present, and GSTP1 IIe105Val polymorphisms and clinical response to chemotherapy and treatment outcome of breast cancers patients.

\section{MATERIAL AND METHODS}

\section{Patients, treatments, and clinical variables}

A total of 310 consecutive patients diagnosed with breast cancer were selected from the Affiliated Hospital of Inner Mongolia Medical University between January 2009 and January 2010. Inclusion criteria for breast cancer patients were female, aged $\geq 18$ years, measurable disease per Response Evaluation Criteria in Solid Tumors; Eastern Cooperative Oncology Group (ECOG) performance status of 0-2; adequate bone marrow, liver, and renal function; and estimated life expectancy at least 3 months.

All patients received chemotherapy. Tumor responses were assessed by contrasted computed tomography scan and/or magnetic resonance imaging every two cycles. Tumor responses were divided into good response and poor response. Patients showed complete re- 
sponse (CR) and partial response (PR) were considered as good responders, and those presented stable disease (SD) and progressive disease (PD) were regarded as poor responders. Overall survival (OS) was calculated from the date of entering study to the date of death from any causes or last clinical follow-up.

All patients received chemotherapy after surgery. The demographic and clinical data of the patients were collected from their medical records. The patients were followed up every four weeks by telephone or attending the clinics, until death or the end of the study.

Written informed consents were provided by all patients, and our study was approved by the ethics committee of the Affiliated Hospital of Inner Mongolia Medical University.

\section{Genotyping}

Prior to the start of chemotherapy, $5 \mathrm{~mL}$ of peripheral blood was taken from each patient for genomic DNA using a Qiagen Blood Kit (Qiagen, Chatsworth, CA, USA) according to manufacturer instructions. The extracted DNA samples were stored at $-80^{\circ} \mathrm{C}$ before genotyping. Genotyping of GSTP1 rs1695, GSTT1 deletion, and GSTM1 deletion was performed by polymerase chain reaction restriction fragment length polymorphism (PCR-RFLP) assay. DNA was amplified through PCR with primers designed with Assay Designer 4.0 (Sequenom, Inc., San Diego, CA, USA). To verify the results, $5 \%$ of the DNA samples were randomly selected for duplicate assays.

\section{Statistical analysis}

Continuous variables are reported as means $\pm \mathrm{SE}$, while categorical variables were shown as frequencies and percentages (\%). The association between GSTP1 rs1695, GSTT1 and GSTM1 polymorphisms and response to chemotherapy in breast cancer patients was assessed using odds ratios (OR) and corresponding 95\% confidence intervals (CIs) in logistic regression analysis. The association between GSTP1 rs1695, GSTT1 and GSTM1 polymorphisms and overall survival of breast cancer patients was evaluated by Hazard ratio (HR) and 95\% CI in Cox Hazard Proportional Model. Kaplan-Meier method was used to curve the overall survival of breast cancer patients. The SPSS software version 16.0 (SPSS Inc., Chicago, IL, USA) for Windows ${ }^{\circledR}$ was used to conduct the analyses. All tests were two-tailed, and values of $\mathrm{P}<0.05$ were considered to be statistically significant.

\section{RESULTS}

The follow-up period of all patients ranged from 2 months to 60 months. Of 310 patients, 170 patients $(54.84 \%)$ were above 50 years old, and $204(65.81 \%)$ were at post-menopause, $6(1.94 \%)$ had a family history of breast cancer, $116(37.42 \%)$ had clinical tumor size $>5 \mathrm{~cm}, 104(35.55 \%)$ had III tumor grade, $128(41.29 \%)$ showed positive estrogen receptor (ER) and 101 (32.58\%) showed positive progesterone receptor (PR) (Table 1).

One hundred and seventy two patients showed a good response to chemotherapy by May 2014, with a good response rate of 55.48\%. After adjusting for potential confounding factors, we found that patients with GG genotype of GSTP1 IIe105Val were more likely to have a poorer response to chemotherapy when compared with those with AA genotype, and the OR $(95 \% \mathrm{CI})$ was $0.37(0.18-0.74)$ (Table 2$)$. Moreover, patients with null genotype of GSTM1 
was associated with a poorer response to chemotherapy than those with present genotype, and the the $\mathrm{OR}(95 \% \mathrm{CI})$ was $0.59(0.36-0.97)$. However, we did not find any association between GSTT1 polymorphism and response to chemotherapy among the breast cancer patients.

By May 2014, a total of 78 patients $(25.16 \%)$ had died. The median overall survival time of breast cancer patients was $42.7 \pm 11.7$ months. By the Cox proportional hazards model, patients with the GG genotype of GSTP1 IIe105Val and null genotype of GSTM1 were found to be correlated with shorter overall survival of breast cancer than homozygotes of the most frequent genotype; the adjusted $\mathrm{HRs}(95 \% \mathrm{CI})$ were $2.51(1.17-5.32)$ and $2.00(1.15-3.48)$, respectively (Table 3 ). However, we did not find any significant association between GSTT1 polymorphism and the overall survival of breast cancer.

\section{Table 1. Characteristics of the study population.}

\begin{tabular}{|c|c|c|}
\hline Characteristics & No. of cases $(\mathrm{N}=310)$ & $\%$ \\
\hline \multicolumn{3}{|l|}{ Mean age, years } \\
\hline$<50$ & 140 & 45.16 \\
\hline$\geq 50$ & 170 & 54.84 \\
\hline \multicolumn{3}{|l|}{ Menopause status } \\
\hline Pre-menopause & 106 & 34.19 \\
\hline Post-menopause & 204 & 65.81 \\
\hline \multicolumn{3}{|c|}{ Family history of breast cancer } \\
\hline No & 304 & 98.06 \\
\hline Yes & 6 & 1.94 \\
\hline \multicolumn{3}{|c|}{ Clinical tumor size $(\mathrm{cm})$} \\
\hline$<2$ & 45 & 14.52 \\
\hline $2-5$ & 149 & 48.06 \\
\hline$>5$ & 116 & 37.42 \\
\hline \multicolumn{3}{|l|}{ Tumor grade } \\
\hline I & 32 & 10.32 \\
\hline II & 174 & 56.13 \\
\hline III & 104 & 33.55 \\
\hline \multicolumn{3}{|l|}{ Estrogen receptor } \\
\hline Positive & 128 & 41.29 \\
\hline Negative & 182 & 58.71 \\
\hline \multicolumn{3}{|c|}{ Progesterone receptor } \\
\hline Positive & 101 & 32.58 \\
\hline Negative & 209 & 67.42 \\
\hline \multicolumn{3}{|c|}{ Response to chemotherapy } \\
\hline Yes & 172 & 55.48 \\
\hline No & 138 & 44.52 \\
\hline
\end{tabular}

Table 2. Correlation between GSTs polymorphisms and tumor response in breast cancer.

\begin{tabular}{|c|c|c|c|c|c|c|c|c|c|}
\hline \multirow[t]{2}{*}{ Genotype } & & \multirow[t]{2}{*}{ Patients } & \multirow[t]{2}{*}{$\%$} & \multicolumn{4}{|c|}{ Tumor response } & \multirow[t]{2}{*}{ OR $(95 \% \mathrm{CI})^{1}$} & \multirow[t]{2}{*}{$P$ value } \\
\hline & & & & Good & $\%$ & Poor & $\%$ & & \\
\hline \multirow[t]{3}{*}{ GSTP1 IIe105Val } & AA & 120 & 38.71 & 76 & 44.19 & 44 & 31.88 & 1.0 (Ref.) & - \\
\hline & $\mathrm{AG}$ & 131 & 42.26 & 73 & 42.44 & 58 & 42.03 & $0.73(0.43-1.25)$ & 0.22 \\
\hline & GG & 59 & 19.03 & 23 & 13.37 & 36 & 26.09 & $0.37(0.18-0.74)$ & 0.002 \\
\hline \multirow[t]{2}{*}{ GSTT1 } & Present & 129 & 41.61 & 76 & 44.19 & 53 & 38.41 & 1.0 (Ref.) & - \\
\hline & Null & 181 & 58.39 & 96 & 55.81 & 85 & 61.59 & $0.79(0.48-1.27)$ & 0.3 \\
\hline \multirow[t]{2}{*}{ GSTM1 } & Present & 190 & 61.29 & 116 & 67.44 & 74 & 53.62 & 1.0 (Ref.) & - \\
\hline & Null & 120 & 38.71 & 56 & 32.56 & 64 & 46.38 & $0.59(0.36-0.97)$ & 0.03 \\
\hline
\end{tabular}

${ }^{1}$ Adjusted for age, menopause status, family history of breast cancer, clinical tumor size, tumor grade, ER and PR. 


\begin{tabular}{|c|c|c|c|c|c|c|c|}
\hline \multirow[t]{2}{*}{ Genotype } & & \multicolumn{2}{|c|}{ Events } & \multicolumn{2}{|c|}{ Alive } & \multirow{2}{*}{$\begin{array}{c}\mathrm{OS}^{1} \\
\text { HR }(95 \% \mathrm{CI})^{1}\end{array}$} & \multirow[b]{2}{*}{$P$ value } \\
\hline & & $\mathrm{N}=78$ & $\%$ & $\mathrm{~N}=232$ & $\%$ & & \\
\hline \multirow[t]{3}{*}{ GSTP1 IIe105Val } & AA & 23 & 29.49 & 97 & 41.81 & 1.0 (Ref.) & - \\
\hline & $\mathrm{AG}$ & 33 & 42.31 & 98 & 42.24 & $1.42(0.75-2.73)$ & 0.25 \\
\hline & GG & 22 & 28.21 & 37 & 15.95 & $2.51(1.17-5.32)$ & 0.01 \\
\hline \multirow[t]{2}{*}{ GSTT1 } & Present & 31 & 39.74 & 98 & 42.24 & 1.0 (Ref.) & - \\
\hline & Null & 47 & 60.26 & 134 & 57.76 & $1.11(0.64-1.94)$ & 0.7 \\
\hline \multirow[t]{2}{*}{ GSTM1 } & Present & 38 & 48.72 & 152 & 65.52 & 1.0 (Ref.) & - \\
\hline & Null & 40 & 51.28 & 80 & 34.48 & $2.00(1.15-3.48)$ & 0.01 \\
\hline
\end{tabular}

${ }^{1}$ Adjusted for age, family history of breast cancer, clinical tumor size, clinical lymph node, and metastasis.

\section{DISCUSSION}

Our study evaluated the association between GSTP1 IIe105Val, GSTT1, and GSTM1 polymorphisms and breast cancer patients' response to chemotherapy and overall survival. Our study suggests that the polymorphisms in GSTP1 IIe105Val and GSTM1 can influence the response to chemotherapy and overall survival of breast cancer patients. The GSTP1 IIe105Val and GSTM1 gene polymorphisms could be helpful to design individualized therapy for breast cancer patients.

GSTs can result in drug tolerance mainly through two pathways. First, directly disintoxication through binding to lipophilic cytotoxic medications to increase the water-solubility and the drainage, and catalyzing the binding of electrophilic materials to glutathione (GSH) (Cnubben et al., 1998). Second, functioning as inhibitor of mitogen-activated protein kinase (MAPK); the GSTs mediate the metabolism of antitumor drugs neither through binding to GSH nor as the enzymatic substrates, but through the interaction with MAPK signaling pathway (Strange and Fryer, 1999). GSTs can be associated with JNK and induced apoptosis, and thus cause tolerance to antitumor chemotherapy drugs.

Recently, many studies indicated that genetic polymorphisms involving GSTM1, GSTP1, and GSTT1 have gained importance in the study of chemotherapy outcome of several kinds of cancers, such as cervical cancer, colorectal cancer, acute lymphoblastic leukemia and lung cancer (Sreeja et al., 2008; Suneetha et al., 2011; Kap et al., 2014). Kap et al. (2014) reported that patients with the present genotype GSTM1 had a poorer survival of colorectal cancer after treatment with oxaliplatin than those without the genotype. In another study in India, Suneetha et al. (2011) showed GSTP1 (Ile/Val) polymorphism has a role in the susceptibility to acute lymphoblastic leukemia and also influence treatment outcome. Another from India, found a significant association between the GSTT1 null genotype with shorter lung cancer survival (Sreeja et al., 2008). Several previous studies reported the association between GSTs polymorphisms and overall survival of breast cancer. Bai et al. (2012) reported a significant association was found between GSTM1 and GSTP1 gene polymorphisms and clinical outcomes in breast cancer cases. Romero et al. (2012) found that GSTP1 may influence the survival of breast cancer patients treated with chemotherapy. Moreover, Zhang et al. (2011) reported that GSTP1 polymorphism was associated with the chemotherapy response in breast cancer. However, Khedhaier et al. (2003) did not find that GSTs gene polymorphisms may predict the clinical response to chemotherapy and overall survival of breast cancer patients. These inconsistencies in results might be due to differences in ethnicities, source of patients, 
disease stages, and/or sample size.

There are two limitations in this study. First, the study subjects were selected from one hospital, and thus they will not better represent all the breast cancer patients. Second, due to limited sample size, the present initial study only two drug metabolizing enzyme which are GSTP1 and GSTM1, associated with significantly shorter overall survival of breast cancer patients. This finding merits large sample size study to validate our results and screen more genetic polymorphisms.

In summary, our findings provided statistical evidence that the variants of GSTP1 and GSTM1 polymorphisms could influence the response to chemotherapy and overall survival in breast cancer patients treated with chemotherapy. The result should prompt to perform a larger prospective study to test the prognostic significance of GSTs gene polymorphisms in breast cancer patients.

\section{REFERENCES}

Bai YL, Zhou B, Jing XY, Zhang B, et al. (2012). Predictive role of GSTs on the prognosis of breast cancer patients with neoadjuvant chemotherapy. Asian Pac. J. Cancer Prev. 13: 5019-5022.

Cnubben NH, Rommens AJ, Oudshoorn MJ and Van Bladeren PJ (1998). Glutathione-dependent biotransformation of the alkylating drug thiotepa and transport of its metabolite monoglutathionylthiotepa in human MCF-7 breast cancer cells. Cancer Res. 58:4616-4623.

Duggan C, Ballard-Barbash R, Baumgartner RN, Baumgartner KB, et al. (2013). Associations between null mutations in GSTT1 and GSTM1, the GSTP1 Ile(105)Val polymorphism, and mortality in breast cancer survivors. Springerplus 2: 450.

International Agency for Research on Cancer. Lung Cancer (2012). Estimated Incidence, Mortality and Prevalence Worldwide in 2012. http://globocan.iarc.fr/Pages/fact_sheets_cancer.aspx. Accessed 2014-1-1.

Kap EJ, Richter S, Rudolph A, Jansen L, et al. (2014). Genetic variants in the glutathione $S$-transferase genes and survival in colorectal cancer patients after chemotherapy and differences according to treatment with oxaliplatin. Pharmacogenet. Genomics. 24:340-347.

Khedhaier A, Remadi S, Corbex M, Ahmed SB, et al. (2003). Glutathione $S$-transferases (GSTT1 and GSTM1) gene deletions in Tunisians: susceptibility and prognostic implications in breast carcinoma. Br. J. Cancer 89:1502-1507.

Mishra A, Chandra R, Mehrotra PK, Bajpai P, et al. (2011). Glutathione $S$-transferase M1 and T1 polymorphism and response to neoadjuvant chemotherapy (CAF) in breast cancer patients. Surg. Today 41: 471-476.

Oliveira AL, Rodrigues FF, Santos RE, Aoki T, et al. (2010). GSTT1, GSTM1, and GSTP1 polymorphisms and chemotherapy response in locally advanced breast cancer. Genet. Mol. Res. 9: 1045-1053.

Oliveira AL, Oliveira Rodrigues FF, Dos Santos RE, Rozenowicz RL, et al. (2014). GSTT1, GSTM1, and GSTP1 polymorphisms as a prognostic factor in women with breast cancer. Genet. Mol. Res. 13: 2521-2530.

Romero A, Martín M, Oliva B, de la Torre J, et al. (2012). Glutathione $S$-transferase P1 c.313A > G polymorphism could be useful in the prediction of doxorubicin response in breast cancer patients. Ann. Oncol. 23: 1750-1756.

Sreeja L, Syamala V, Hariharan S, Syamala VS, et al. (2008). Glutathione $S$-transferase M1, T1 and P1 polymorphisms: susceptibility and outcome in lung cancer patients. J. Exp. Ther. Oncol. 7: 73-85.

Strange RC and Fryer AA (1999). The glutathione $S$-transferases: influence of polymorphism on cancer susceptibility. IARC. Sci. Publ. 148: 231-249.

Suneetha KJ, Nancy KN, Rajalekshmy KR, Rama R, et al. (2011). Role of glutathione-s-transferase and CYP1A1*2A polymorphisms in the therapy outcome of south Indian acute lymphoblastic leukemia patients. Indian. J. Med. Paediatr. Oncol. 32:25-29.

Townsend DM and Tew KD (2003). The role of glutathione-S-transferase in anti-cancer drug resistance. Oncogene 22: 7369-7375.

Tulsyan S, Chaturvedi P, Agarwal G, Lal P, et al. (2013). Pharmacogenetic influence of GST polymorphisms on anthracycline-based chemotherapy responses and toxicity in breast cancer patients: a multi-analytical approach. Mol. Diagn. Ther. 17: 371-379.

Zhang BL, Sun T, Zhang BN, Zheng S, et al. (2011). Polymorphisms of GSTP1 is associated with differences of chemotherapy response and toxicity in breast cancer. Chin. Med. J. 124: 199-204. 